\title{
A BRIEF ANALYSIS ON VOCABULARY LEARNING STRATEGIES IN SECOND LANGUAGE 浅谈二语词汇学习策略
}

\author{
Temmy \\ Chinese Department, Faculty of Humanities, BINUS University \\ Jl. Kemanggisan Ilir III No. 45, Kemanggisan-Palmerah, Jakarta Barat 11480 \\ temmy@binus.edu
}

\begin{abstract}
This article tries to explore the usage of vocabulary learning strategies in second language learning on students with different background. It attempts to elicit useful ideas and gives a practical guidance in learning Chinese as a second language. This work explains how learning environment, cultural background and proficiency level can highly affect the choice of learning strategies. And even though students are learning different languages, there are similarities in their choice of strategies during the process of acquiring the target language.
\end{abstract}

Keywords: Vocabulary ，L2, Learning environment, Cultural background, Proficiency level

\section{内容提要}

本文试图探讨不同背景的二语学习者在词汇学习策略方面的使用，以期获得有关二语词汇学习策略 方面的有益启示, 并能更好地促进词汇学习与教学。本文发现学习环境、文化背景和语言水平是导致学 生在二语词汇学习策略使用差异的因素。本文发现虽然学习者所学习的二语语种不一样, 不过他们的成 功之道具有一些共同之处。

关键词 : 词汇二语学习环境文化背景语言水平 


\section{前言}

不同背景的学生在二语词汇学习策略的使用方面虽然有着某些相似之处，但更多表现出的 还是差异。那么, 究竟是哪些因素导致了这些差异的产生呢? 本文中, 笔者将从学习环境、文 化背景和语言水平等方面来做个简单的分析, 以期更深入地探讨这些因素对词汇学习策略使用 的影响。

\section{内容}

\section{学习环境与词汇学习策略}

语言的学习环境对于学习策略的影响已经引起了很多研究者的注意, 已有相关研究指出学 习者在目的语环境与在非目的语环境中, 所采用的学习策略是不同的。而在本研究中, 学习环 境对学生学习二语词汇时使用学习策略的影响主要表现在以下几个方面：

\section{- . 学习环境与词汇学习的资源应用策略}

在目的语学习环境中的学生 (例如来中国学习汉语的外国留学生) 比在非目的语学习环境 中的学生 (例如在中国学习英语的中国大学生) 会更经常、也更好地使用资源应用这一策略。 目的语的学习环境为学习者提供了大量真实的学习资源，从而在客观上有力地促使学习者利用 这一社会环境的资源，在社会交际中进一步学习语言知识，这一点是母庸置疑的。据调查，在 众多的资源应用策略中受学习环境影响最明显的当属寻找语言伙伴这一条子策略了。目的语学 习环境为学习者提供了非常难得而珍贵的寻找语言伙伴的平台。之前吴勇毅教授在 2008 年发表 的《意大利学习口语交际策略的个案分析》中也指出学习者在目的语环境中使用学习策略时非 常突出的便是语言伙伴这一策略。由此可见，这两者之间的相关性是有一定的普及性和科学性 的。 


\section{二. 学习环境与词汇学习的认知策略}

学习环境对于学习者认知策略的使用也有一定的影响, 其中最明显的就是学习者的查词典 策略。

目的语学习环境中的学习者 (例如来中国学习汉语的外国留学生) 比非目目的语环境中的 学习者(例如在中国学习英语的中国大学生)更加积极地使用查词典策略。目的语的学习环境为学 习者提供了沉浸式的交际环境, 学习者无时无刻都有需要利用所学的目的语词汇进行交际的机 会。这样一来, 接触到新词汇或是巩固已学词汇的几率大大提高, 从而也从客观上使学习者查 词典的需求大量产生。这一点是可以理解的, 并且对于学习者的词汇学习有百利而无一害。

\section{三. 学习环境与词汇学习的元认知策略}

除了资源应用和认知策略受到学习环境的影响之外, 词汇学习的元认知策略也与学习环境 有一定的相关性，特别是在“自我检查”以及“与母语人士比较”这两条子策略上。调查结果表明在 目的语环境中的学习者 (例如来中国学习汉语的外国留学生) 比非目的语环境中的学习者 (例 如在中国学习英语的中国大学生 ) 更加积极地使用上述两条元认知策略。

当学习者在目的语环境中学习时, 自然有更多运用所学词汇的机会, 并且目的语的学习环 境也能够及时地给出学习者相对的反馈，从而也就使学习者有更多检验自己词汇的机会。虽然 一开始的时候学习者未必有意识去主动检验自己的词汇学习情况，但在一次次的反馈之后，学 习者逐渐建立起了自我检查的良好学习意识。 
同样, 当学习者身处目的语学习环境时, 大量接触母语人士的机会使他们比非目的语学习 环境中的学习者有更好的条件可以比对自己和母语人士在用词上的差别。虽然这一策略的使用 在刚开始时也并非是出于自觉, 而是通过与母语人士的交际而自然获得的, 但学习者在这样的 环境中会逐步慢慢建立起使用这一策略的主动性和习惯。

\section{文化背景与词汇学习策略}

学习者的文化背景和他们所使用的词汇学习策略之间也有紧密的联系。不同文化背景的学 习者会采用不同的词汇学习策略。文化背景这一因素对词汇学习策略的影响主要表现在以下几 个方面：

\section{一 . 文化背景与词汇学习的元认知策略}

文化背景对于词汇元认知策略的使用有一定的影响，主要表现在“调整学习方法”这一条子策 略上。外国留学生要比中国大学生更善于使用这一学习策略。

西方文化下的教育体系宽松而自由，从而给予学生很大的自由探索的空间，老师教的未必 就是标准答案。一般外国留学生就在这样探索式的学习环境中摸索学习，遇到困难或是失败之 后，他们自然而然地逐渐学会了调整自己的学习方法和节奏。

而相比较之下, 在讲究“尊师重道”传统中国文化下成长起来的中国大学生所受到的教育则很 不同。他们注重细节的准确性，亦步亦趋跟随老师的步伐，最好一切的问题都有标准的模板可 以用来套答案。在应试教育灌输下的中国大学生尚不能像外国留学生那样从整体上反思自己的 
学习情况, 也没有那么多的机会和空间自由探索。很多大学生表示自己很少考虑“学习方法”的问 题, 即使学习的效果不理想, 也没去思考该如何调整学习方法, 即使觉得自己的方法不适合自 己，也不知道该从何入手做出改变，这一点是值得我国英语教学深思的。

\section{二 . 文化背景与词汇学习的认知策略}

文化背景对于学习者词汇学习的认知策略的使用也有一定的影响, 主要反映在记忆策略上。 其中最明显的是“背诵生词表”以及“做生词卡片或是记录本”这两条子策略上。虽然中国大学生在 观念上已经接受了“通过上下文来记忆词汇”这一观念, 但在实际的学习过程中, 受到中国传统教 育理念熏陶的中国大学生仍然放不下传统的“背诵生词表”的方法。很多大学生表示从小到大，对 于学习英语单词最深刻的印象便是要背诵, 几乎所有的英语教材中都会有附加的生词表, 而且 书店里还有很多五花八门的词汇背诵手册。从这些事例可以看出其实以往在中国传统的教育理 念下, 背诵是一种颇受推崇的学习方法, 这种观念已经深深植入了中国大学生的脑海中。所以 即使观念改革了, 新的词汇学习理念被引进甚至也广泛地被学习者所接受，但是过去观念的影 响力还是非常深刻的。另外, 也是由于中国大学生习惯于背诵“现成”的生词表, 所以很少有人会 自己动手“做生词卡片或是记录本”。而相对的，外国留学生则正好相反，很少有人会“背诵生词表”, 但是却有很多学生会自己动手制作生词卡片（Flashcard）。其实这也是由于受到西方文化下教育 理念的影响。西方的教育理念崇尚“独立思考，质疑一切”的创新精神，而不提倡“死记硬背”等循 规蹈矩的观念。因此西方国家大学生从小接受的教育理念便是由学生自己去探索，因此不会有 什么现成的生词表等着大学生们去背诵。西方国家的学生表示, 从小到大, 不论他们小时候学 习母语英语时，还是长大后学习另外一门语言，例如法语、日语、西班牙语的时候，老师都建 议他们自己动手制作生词卡片。他们觉得这样一个自己动手制作、自己书写、自己设计的过程 
是将原本书本上的生词转变为自己知识的一个很好途径。从这里, 可以明显看到东方和西方文 化背景差异下不同的教育理念对于学习者认知策略使用的影响。

\section{三. 文化背景与词汇学习的社会情感策略}

文化背景对于社会情感策略的影响主要表现在“与他人交流合作”这一子策略上。中国含蓄内 敛的文化熏陶使得中国大学生不太善于和别人交流与分享学习过程中的心得，不太懂得怎样通 过和别人分享、合作来帮助自己的学习。而且, 中国学校的教育模式也很少为学生提供一个分 享交流的平台。这样一来, 学生们更加无从开始学习怎样和别人交流合作。反之, 西方国家开 放的多元文化大背景下, 外国留学生更乐于与他人交流和分享自己的学习感受, 也更懂得如何 通过这样一个合作的过程来帮助自己的学习。西方的教育也非常注重培养学生与他人合作、沟 通、交流的能力, 从小学开始, 不论是何种学科, 团队合作是免不了的任务。而就是在这样一 次次与同学相互合作的过程中，学习者的合作、沟通能力就得到了很大的提高。

\section{四 . 文化背景与词汇学习的资源应用策略}

外国留学生普遍比中国大学生更青睐使用“寻找语言伙伴”这一资源应用策略。虽然这与之前 分析的学习环境之间有很大的关系, 但同时也是由于受到了文化背景因素的影响。中国传统文 化提倡含蓄内敛，因此大多数的中国大学生不太敢主动地去寻找语言伙伴，怕说错或是被别人 拒绝而丢脸。而与他们的英语水平相比, 汉语口语水平整体偏低的西方大学生却更加积极地寻 找伙伴, 即使是初级阶段的学生, 也不害怕自己水平有限无法和语言伙伴交流。因为开放的西 方文化精神的浸润使得来自西方国家大学生大多具备独立、乐于接受挑战和主动探索的精神。 


\section{语言水平与词汇学习策略}

学习者的语言水平的高低同样会影响到词汇的学习策略。学生在词汇学习策略使用方面的 某些差异也是由于他们的语言水平高低不同所致。总的来说, 具体表现在以下几个方面：

\section{一. 语言水平高低与词汇学习的元认知策略}

语言水平高低对于学习者元认知策略的使用有一定的影响，具体主要体现在“检查词汇学习 情况”这一子策略上。语言水平较高的学习者 (例如在中国学习英语的中国大学生) 与语言水平 较低的学习者 (例如来中国学习汉语的外国留学生) 相比, 低水平的语言学习者会更经常地对 自己的词汇学习情况进行检查。语言水平高低与这条策略的使用频率成反比。通过进一步的访 谈，笔者发现当学习者的语言水平还停留在相对较低的程度时,他们相对来说注意的范围有限， 由于词汇对于他们来说是学习的“重中之重”, 因此他们对于自己的词汇掌握情况自然比较关心， 对于检查自己词汇的掌握情况比较重视。而当学习者的语言水平提高了之后, 他们关注的学习 焦点可能会慢慢扩大, 从一开始的类别化的发音标准度，词汇掌握量逐渐发展到口语流利度、 写作流畅度等更高的综合性学习层面上, 这个时候学习者投入在专门检查词汇学习情况上的时 间就会减少。

\section{二 . 语言水平高低与词汇学习的认知策略}

学习者语言水平的高低与其认知策略的使用情况之间也有很多的关联, 主要表现在以下几 个方面: 首先是查词典策略上, 语言水平较高的学习者（例如在中国学习英语的中国大学生） 与语言水平较低的学习者 (例如来中国学习汉语的外国留学生) 相比, 语言水平较高的学习者 在查字典时更加注重词的搭配和用法, 而语言水平较低的学习者在查字典时则更加注重词的发 
音和意思。这一点是由于语言水平不同的学习者所面对的外部学习任务要求不同所引起的。诸 如整体汉语水平较低的外国大学生所面对的一些学习任务相对来说综合性的要求不高, 所以他 们在查词典时的心理期待便是词的发音和意思。而整体英语水平较高的中国大学生所面临的学 习任务相对来说综合性要求较高, 所以他们在查词典时更加注重词的搭配和用法。此外, 有很 多英语词汇有一系列不尽相同的意思, 较高水平的学习者可能已经掌握了其中一个常用意义, 可是还有更高要求的学习需要, 而这时当然也就更关注该词新意义的搭配和用法。其次, 记笔 记策略也受到语言水平的影响，笔者发现语言水平较低的学习者（例如来中国学习汉语的外国 留学生 ) 要比语言水平较高的学习者 (例如在中国学习英语的中国大学生 ) 更偏爱使用记笔记 策略。进一步的访谈发现这可能是由于语言水平较低的学习者尚不具备选择性注意的能力, 他 们不知道什么是重点和难点, 哪些内容对他们来说比较重要, 所以他们对于记笔记比较重视, 并且容易全盘照抄。而对于那些语言水平较高的学习者来说，他们已经历过勤勤既恳记笔记的 那段时间，现在他们已经具备了选择注意自己所需要的重点的能力，所以他们记笔记的量自然 减少。但这从另一个方面来说其实也是一个好事情。最后, 与语言水平较高的学习者相比, 语 言水平较低的学习者更重视复习，但同时对于母语的依赖度也更高。语言水平较低时，学习者 脑中尚还不能将目的语的第二信号系统直接快速地与第一信号系统项联系, 而是要通过母语的 第二信号系统来进行中转。而当语言水平达到一定程度之后, 将目的语的第二信号系统与第一 信号系统直接相连的能力增强，那时对于母语的依赖程度也就自然而然降低了。

\section{三．语言水平高低与词汇学习的资源应用策略}

学习者语言水平的高低与其在资源应用策略方面的使用情况也有密切的联系。应该说, 从 总体上来看, 语言水平较高的学习者 (例如学习英语的中国大学生) 相对于语言水平较低的学 习者 (例如学习汉语的外国留学生) 而言, 在资源应用策略方面的使用率要高。 
具体而言, 语言水平的高低对以下两条资源应用子策略的影响最大：听目的语广播和看目 的语电视、阅读目的语报纸和杂志书籍。语言水平较高的学习者往往在这两条资源应用子策略 方面的使用率要高于语言水平较低的学习者。这一点也是可以理解的, 毕竟这两条子策略对于 学习者的语言综合能力要求较高, 只有当学习者的语言水平达到一定的高度之后, 运用起来才 能较为得心应手, 进而才能促进他们的学习, 增强利用这些资源来帮助自己学习的动力。而那 些语言水平较低的学习者即使有心利用这些学习资源来帮助自己的词汇学习, 但是由于语言水 平和实际运用之间的差距实在太大，在运用失败之后，这些学习者会无可避免地产生挫败感， 从而在日后逐渐不再使用这些策略。

\section{小结}

本文发现在二语学习的过程中, 虽然语种不一样, 但能够达到高水平的学习者都具 有一些共同之处。例如他们都非常重视词汇学习, 注重元认知策略的使用。他们也采用 多种不同的记忆方法, 并认为目的语输入对于词汇学习有着不可忽视的积极意义, 并能 够积极利用各种目的语资源帮助自己学习词汇。

\section{参考文献}

徐子亮.对外汉语学习理论研究二十年[J].《世界汉 语教学》, 2004(4).

吴勇毅. 学习策略对汉语作为第二语言学习者的影响 $[\mathrm{M}]$. 对外汉语教学的跨文化视角, 上海: 华 东师范大学出版社, 2004.

Chamot A.U. (1987). The learning strategies of ESL students. InA.Wenden\&J.Rubin (Eds.). Learning strategies in language learning . PrentieeHall. 
Cohen, A. D. (2000). Strategies in Learning and Using a Second Language. 北京 : 外语教学 与研究 出版社.

Coady, J., \& Huckin, T. (2001). Second Language Vocabulary Acquisition. [M] 上海：上海外 语教育 出版社. 